\title{
AN INTEGRATED APPROACH OF ART1 AND TABU SEARCH TO SOLVE CELL FORMATION PROBLEMS
}

\author{
Ming-Laing Chen \\ Department of Industrial Engineering \\ Mississippi State University \\ Chung-Min $\mathrm{Wu}^{*}$ \\ Department of Business Management \\ National Taipei University Technology \\ 1, Section 3, Chung-Hsio East Road, Taipei, Taiwan, 106, R.O.C. \\ Chuen-Lung Chen \\ Department of Management Information Systems \\ National Chengchi University
}

\begin{abstract}
Adaptive resonance theory (ART1) network is one of the many popular neural networks used to solve the cell formation problem. Several modifications of ART1 for the problem have recently been published. In this study, a modified ART1 network is integrated with an effective optimization technique, Tabu Search (TS), to solve cell formation problems. The number of exceptional elements (EE) and group efficiency (GE) are considered as the objectives for the problems under the constraints of the number of cells and cell size. This proposed heuristic (ART1\&TS) first constructs a cell formation using a modified ART1, and then refines the solution using a basic TS heuristic. ART1\&TS has been applied to most popular examples in the literature. The computational results showed that it generated the best solutions in most of the examples.
\end{abstract}

Keywords: adaptive resonance theory (ART1), cell formation, tabu search

\section{INTRODUCTION}

Cellular manufacturing $(\mathrm{CM})$ is an application of group technology (GT) principles to manufacturing. In a cellular manufacturing system, machines are grouped into machine cells and parts are grouped into part families according to the similarities of the product design and manufacturing process. Using this method, manufacturing costs such as setup times, labor, throughput times, material handling times, work-in-process, and tooling can be reduced, and a more effective and productive manufacturing environment can be created.

Cell formation, which generates the groups of machines and parts, is an essential problem when cellular manufacturing is considered. Lots of approaches have been developed to solve the cell formation problem. According to Singh [27], these approaches can be classified into the following categories: coding and classification, machinecomponent group analysis, similarity coefficients, knowledge-based, fuzzy clustering, neural networks, and heuristics. A complete survey of these approaches can be found in Singh [27], and Heragu [17].

Neural network based approaches are the new tools for cell formation problems. The neural network, also called the artificial neural system, is a recent development in artificial intelligence. Unlike nonlearning algorithms, neural models are able to learn and store learned patterns according to their learning disciplines. The Adaptive Resonance Theory (ART1) model-- presented by Carpenter and Grossberg [5]-- is one of the most popular neural network models used for solving cell formation problems. Using this model, a cell formation problem is first presented as a zero-one matrix with machines as rows and parts as columns; an entry of one represents that the corresponding part is processed by the corresponding machine, or zero otherwise. Then the machine rows are used as input vectors to the input layer of ART1, and are grouped into cells by comparing the similarity among the machines and a specified vigilance threshold value.

The theory of ART1 shows that its efficiency is significantly affected by two factors: the order that

*Corresponding author: cmwu@ntut.edu.tw 
the vectors are assigned to its input nodes and the specified vigilance threshold value. Dagli and Huggahalli [12] applied a basic ART1 model to solve cell formation problems. Since then several modifications to the basic model, principally concerning the order of input vectors, have been conducted by Kaparthi and Suresh [18], Rao and Gu [24], Kaparthi, Suresh, and Cerveny [19], and Chen and Cheng [11]. Although these modifications have improved the performance of ART1, the order of the input vectors may still affect its efficiency. It is believed that an application of optimization methods to the solution generated by ART1 should be able to improve the performance ART1 for the candidate problem.

In this research, it is proposed that a modified ART1 is integrated with an effective optimization technique, Tabu Search (TS), to solve cell formation problems. Two common criteria: the number of exceptional elements (EE) and group efficiency (GE) are considered as the objectives. Two practical constraints: number of cells (NG) and upper limit of cell size are used as the constraints. This approach (ART1\&TS) first constructs a cell formation using the modified ART 1, and then refines the solution by using basic TS heuristic. The performance of ART1\&TS will be evaluated by applying it to most common test problems, and comparing its solutions with the best existing solutions.

Figure 1 displays a general solution for a cell formation problem. Each diagonal submatrix refers a machine-cell and its corresponding part family. The 1 's in the off-diagonal submatrices are defined as exceptional elements. Given that [8],

$\mathrm{M}$ : number of machine rows

$\mathrm{N}$ : number of part columns

$e_{d}$ : number of 1's in the diagonal blocks

$e_{o}:$ number of 1's in the off-diagonal blocks

$q$ : weighting factor

$k \quad$ : number of blocks

$G_{r}$ : number of machine rows in the $r$ th block

$C_{r}$ : number of part columns in the $r$ th block

The GE of a solution is defined as follows:

$$
\begin{aligned}
& \mathrm{GE}=q \boldsymbol{\eta}_{l}+(1-q) \boldsymbol{\eta}_{2}, \text { where } \\
& \boldsymbol{\eta}_{\mathbf{1}}=\frac{e_{d}}{\sum_{r=1}^{k} G_{r} C_{r}} \\
& \boldsymbol{\eta}_{2}=1-\frac{e_{o}}{\mathrm{MN}-\sum_{r=1}^{k} G_{r} C_{r}}
\end{aligned}
$$

and $0 \leqq q \leqq 1$. In general, an equal weight $(q=$ $0.5)$ is recommended.

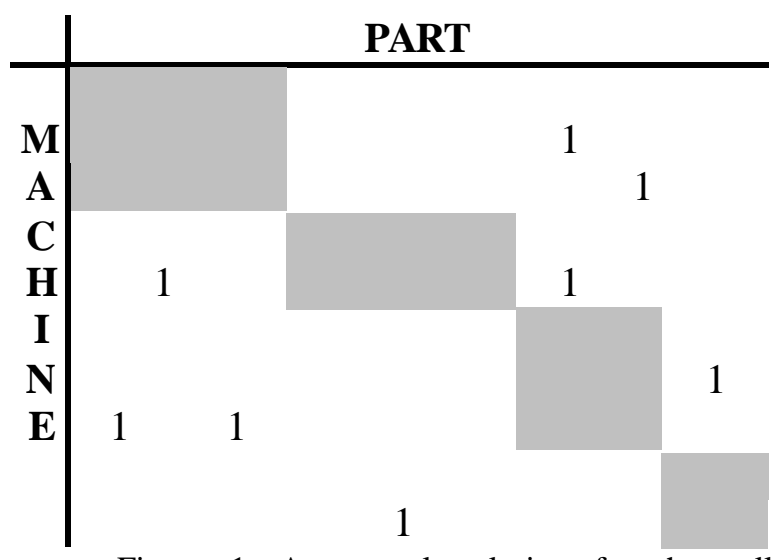

Figure 1. A general solution for the cell formation problem

In the following section a detailed discussion on the application of ART1 to cell formation problems is provided. A brief review of TS is presented in Section 3 , and the ART 1\&TS heuristic is presented in Section 4. In Section 5, a computational result, evaluating the performance of ART1\&TS, is presented and discussed in detail. The major conclusions of this research are provided Section 6.

\section{THE ART1 HEURISTIC AND MODIFICATIONS}

The ART1 network is similar to networks using a single Kohonen layer with competitive learning neurons. It includes two properties of neural networks: plasticity and stability. Plasticity is the capability of the network to learn new things from external information thereby changing its internal connections. Stability is the ability of the network to retain its useful memory of previously stored patterns. Furthermore, ART1 is based on unsupervised learning. That means the network has no knowledge of the correct output. For the cell formation problem, unsupervised learning is suitable because there is no information about correct group formation.

ART1 network contains two layers of neurons: the input and output layers. Every neuron in the input layer is entirely connected to each neuron in the output layer with a bottom-up connection. Every neuron in the output layer is entirely connected to each neuron in the input layer with a top-down connection. The number of neurons in the input layer is equal to the number of components $(\mathrm{N})$ in the input vector, and the number of neurons in the output layer is dependent on the maximum expected number of clusters (C) that the input vectors can be classified into. In general, the first input vector is the exemplar for the first cluster. When the next input vector is presented, it will be compared with the first cluster exemplar. If the similarity coefficient between the vector and the exemplar is larger than a specified 
vigilance threshold value, it will be assigned to the cluster, and the exemplar will be updated. Otherwise, this input vector becomes the exemplar for the second cluster. This process is repeated for all the input vectors. The ARTI algorithm is outlined below ([11] and [18]).

Step 0. Define the number of neurons in the input layer, numbered from 1 to $\mathrm{N}$, and the number of neurons in the output layer, numbered from 1 to $\mathrm{C}$.

Step 1. Initialize the weights and set a vigilance threshold $\rho$.

$\mathrm{t}=0$;

Top-down connection weights: $\mathrm{W}_{\mathrm{ij}}(\mathrm{t})=1$;

Bottom-up connection weights:

$\mathrm{V}_{\mathrm{ij}}(\mathrm{t})=1 /(\mathrm{N}+1)$, with $\mathrm{i}=1, \ldots, \mathrm{N}$ and

$\mathrm{j}=1, \ldots . \mathrm{C} ; 0 \leqq \rho \leqq 1$;

Step 2. Present an input vector $X$ (consisting of binary elements $\mathrm{x}_{\mathrm{i}}$ ) to the input layer.

Step 3. Compute the matching score for every output neuron.

The matching score, $y_{j}$, of output neuron $\mathrm{j}$ is computed as follows.

$y_{j}=\sum_{i=1}^{\mathrm{N}} v_{i j}(t) \cdot x_{i}, \quad 1 \leq j \leq \mathrm{C}$.

Step 4. Select the best matching output neuron, $\mathrm{y}_{\mathrm{j}}{ }^{*}$

$y_{j}^{*}=\max _{1 \leq j \leq C}\left\{y_{j}\right\}$.

In case of tie, select the neuron with the smallest number.

Step 5. Conduct the vigilance test.

$$
|X|=\sum_{i=1}^{N} x_{i}
$$$$
|W \cdot X|=\sum_{i=1}^{N} w_{i j^{*}}(t) \cdot x_{i},
$$

If $\frac{|W \cdot X|}{|X|}>\rho$, go to Step 7;

otherwise, go to Step 6.

Step 6. Deactivate the best matching output neuron. The score of best matching neuron selected in Step 4 is temporarily set to zero. That is this neuron will not be considered in the current cluster search. Go to Step 3

Step 7. Update the weights associated with the best matching output neuron.

$$
\begin{aligned}
& \left.\mathrm{W}_{\mathrm{ij} *}(\mathrm{t}+1)=\mathrm{W}_{\mathrm{ij} *}(\mathrm{t})\right] \mathrm{x}_{\mathrm{i}}, \text { and } \\
& v_{i j^{*}}(t+1)=\frac{w_{i j^{*}}(t) \cdot x_{i}}{0.5+\sum_{i=1}^{N} w_{i j^{*}}(t) \cdot x_{i}},
\end{aligned}
$$

for $\mathrm{i}=1$ to $\mathrm{N}$.

Step 8. Activate the neurons deactivated in Step 6; go to Step 2.

To apply the ART1 to a cell formation problem, the problem is described as a zero-one matrix with machines as rows and parts as columns, and each row (machine) is used as an input vector to the input layer of the ART1. The similarity coefficient, | W $\mathrm{X} \mid$ $/|X|$, in step 5 determines the degree of match between an input vector (X) and a cluster exemplar (W). When implementing ART1, the threshold value significantly affects the result. When a high value of $\rho$ is set, a high degree of match within each cluster is commanded and the input vectors are classified into a large number of clusters. On the other hand, if a low value of $\rho$ is used, the input vectors are classified into a small number of clusters.

Another factor that will affect the performance of ART1 is the updating operation of the top-down connection weights $\mathrm{W}_{\mathrm{ij}}$ (the exemplar of output neuron $\mathrm{j}$ ) in Step 7 . With this operation, $\mathrm{W}_{\mathrm{ij}}(\mathrm{t}+1)$ can be 1 only if both $\mathrm{W}_{\mathrm{ij}}(\mathrm{t})$ and $\mathrm{X}_{\mathrm{i}}$ are 1 , which leads to a result that a new exemplar always has a lower number of 1's than that of old exemplars. This will cause misclustering because 1) a new input vector, which has a high similarity with the newest exemplar of an output neuron, may have a low similarity with the vectors that have been assigned to the neuron, or 2) a new input vector, which has a low similarity with the newest exemplar of an output neuron, may have a high similarity with the vectors that have been assigned to the neuron. Furthermore, since a new exemplar is determined by the current exemplar and the first qualified input vector followed, the order that the input vectors are presented to ART1 will significantly affect the output of ART1.

Research has been conducted to solve these problems. Dagli and Huggahalli [12] suggested the input vectors be presented to ART1 in descending order of the number of l's in each vector. Kaparthi and Suresh [18] suggested that the binary entries in the machine-part matrix be reversed. The density of 1 's in the machine-part matrix for a cell formation problem is generally low. Reversing the entries in the matrix, and presenting the input vectors in descending order of the number of l's in each vector may increase the possibility of bringing the similar vectors together before presenting them to ART1. This reduces the possibility of misclustering. However, Chen and Cheng [11] showed that both methods did not result in reasonable solutions. 
Rao and $\mathrm{Gu}[24]$ addressed the following method to update the top-down connection weights. The input vectors are presented to ART1 in descending order of the number of l's in each vector. If an input vector is qualified to be assigned to an output neuron $\mathrm{j}$, the top-down connection weights of neuron $\mathrm{j}$ will be updated as follows: $\mathrm{W}_{\mathrm{ij}}(\mathrm{t}+1)$ is equal to 1 as long as $\mathrm{W}_{\mathrm{ij}}(\mathrm{t})$ or $\mathrm{X}_{\mathrm{i}}$ is equal to1; otherwise, $\mathrm{W}_{\mathrm{ij}}(\mathrm{t}+1)$ is equal to 0 . When implementing this method, the number of l's in the new exemplar will be no less than that in the old exemplars.

This method did not completely solve the misclustering problems of ART1; however, it improves the performance of ART1.

Chen and Cheng [11] developed a rearrangement algorithm to determine the order of presenting the input vectors to ART1. This algorithm is implemented in the following steps: 1) apply ART1 to generate the initial machine-part groups; 2) in each machine-part group identify the machine vectors which have more number of l's outside the group than the number of l's inside the group, and set these vectors aside; 3 ) in each machine-part group rearrange the machine vectors in descending order of the number of l's in the group; 4) group and rearrange the vectors set aside in the second step in descending order of the number of l's in the vectors. After implementing this algorithm, the machine vectors are again presented to ART1 in the order generated by the last two steps of the algorithm, and this produces a new machine-part groups.

There are two shortcomings when applying this algorithm to cell formation problems. First, the solution may still be influenced by the order of the vectors presented to ART 1. Second, it is difficult to determine a suitable value of $\rho$ for ART1. In this paper, Chen and Cheng tried different $\rho$ values from 0 to 0.99 with an increment of 0.01 . In order to further improve the solution generated by the rearrangement algorithm, a simple reassignment algorithm was developed in the same paper. This algorithm identifies the bottleneck machine vectors in the machine-part groups generated by the rearrangement algorithm, and reassigns each of them to the machine cell in order to reduce the exceptional points. For some problems, these two algorithms have to be implemented more than once. This makes the application of ART1 tedious.

As mentioned previously, in this research a heuristic (ART1\&TS) integrating ART1 and TS has been developed for cell formation problems. To implement ART1\&TS, ART1 with the modification of Rao and Gu (denoted as Modified ART1), due to its simplicity, is used to generate the initial cell formation. TS is then used to refine the formation. The procedure of TS is to iteratively move machine vectors from cell to cell so as to improve the formation (see the next section for detailed discussion). The critical factor that may affect the efficiency of ART1\&TS is the determination of the vigilance threshold value while implementing Modified ART1. If the value is large, Modified ART1 may yield a formation with a large number of cells. This causes the work to be tedious when implementing TS to improve the formation. If the value is small, Modified ART 1 usually yields a formation with a small number of cells and some of them filled up. This may interfere moving machine vectors among the cells, and may affect the performance of TS.

To overcome this flaw, a method for determining the maximum number of cells -- before implementing Modified ART 1-- has been developed. According to a specified upper limit of cell size $(\mathrm{m})$ for a problem, it first uses a coefficient, $\delta$ (denoted as a scale coefficient), to determine the maximum number of machines allowed in each cell for Modified ART1, where $0 \leqq \delta \leqq 1$. For instance, if the upper limit of cell size is $\mathrm{m}$, the maximum number of machines allowed to be assigned to each cell is equal to $\mathrm{m} \delta$ (rounding up if the value is not an integer). The maximum number of cells that the machines in the problem can be clustered into is then determined by dividing the number of machines (M) by $\mathrm{m} \cdot \delta$ (rounding up if the value is not an integer). This maximum number of cells is then used as the number of output neurons for Modified ART1. Each of the machines that could not be clustered into any of the output neurons, after implementing Modified ART 1, will be assigned to the output neuron that will result in the smallest number of exceptional elements. Since the previous procedure will generate the initial solution with a reasonable number of cells (each cell is not filled completely), it is believed that the efficiency and effectiveness of using TS to improve the initial solution will be enhanced.

\section{TABU SEARCH}

Tabu search (TS) is a meta-heuristic developed by Glover [14]. Since its introduction, TS has been successfully applied to a wide variety of research areas such as scheduling, designing, routing, location and allocation, logic and artificial intelligence, technology, telecommunications, production, inventory and investment, graph optimization, and general combinatorial optimization [15].

The main idea of TS is to guide a search out of local optimality by accepting the neighboring solutions that are not better than the corresponding current solution. Like other neighborhood search techniques, TS starts with an initial solution (the current solution, as well as the incumbent solution) and evaluates the objective values of its neighboring solutions. The best solution in the neighborhood will 
be chosen, and used to replace the current solution, even though it may not be better than the current solution. If the current solution is better than, or equal to the incumbent solution, the incumbent solution is updated with the current solution. A move is defined as the search that leads to the exchange of the current solution for the neighboring solution. To prevent cycling, and to force the search to new solution regions, a move that has been chosen to produce a new solution will be immediately assigned to a list termed tabu. This move will not be chosen in a number of iterations followed. This number of iterations is denoted as the tabu list size. Once the tabu list has reached its specified size, the move that has been placed in the list earliest is removed as the most current move is inserted. The search process continues until certain termination criteria are met.

According to previous research $[14,30,31]$, the performance of TS was found to be influenced by the following factors: the initial solution, the type of move, the neighborhood size, the tabu list size, and the termination criterion.

\subsection{Initial Solution}

The initial solution, in TS applications, can either be a random or a heuristic solution [31]. A good initial solution may lead to a better final solution. In this research, for a cell formation problem, a random initial solution was generated by randomly and equally assigning machines to the specified maximum number of cells of the problem. A heuristic initial solution was generated by using Modified ART1.

\subsection{Type of Move}

A step that modifies the current solution is called a move, and the solution generated by the move is called a neighboring solution of the current solution. As mentioned previously, for cell formation problems, a solution is presented as a zero-one matrix with machines as rows and parts as columns. There are two common methods to modify a solution [31]. One is to swap two machine rows in different cells, denoted as SWAP; the other is to choose a machine row from one cell and insert it in another cell, denoted as INSERT. For instance, a cell formation problem includes $\mathrm{M}$ machines and $\mathrm{N}$ parts. Let $\mathrm{M}$ be an even number. Without loss of generality, let us construct an $\mathrm{M} \times \mathrm{N}$ matrix in order of the machine number, and generate a solution $X$ by assigning the first half of the machines to cell 1 and the second half of the machines to cell 2 . The solution $X$ can be presented as a column vector,

$$
X=\left[\begin{array}{c}
x_{1} \\
x_{2} \\
\vdots \\
x_{(M / 2)} \\
x_{(M / 2)+1} \\
x_{(M / 2)+2} \\
\vdots \\
x_{M}
\end{array}\right]
$$

where $x_{i}$ is the row vector of machine $i$ in the $\mathrm{M} \times \mathrm{N}$ matrix.

If a move is to swap the first machine in cell 1 and the first machine in cell 2 , then the transpose of the solution generated by the move is $\mathrm{X}_{\text {new }}=\left[x_{(M / 2)+1}\right.$, $\left.x_{2}, x_{3}, \ldots, x_{(M / 2)}, x_{1}, x_{(M / 2)+2}, \ldots, x_{M}\right]$. On the other hand, if a move is to insert the first machine in cell 1 to the position between the first and second machines in cell 2 , then the transpose of the solution generated by the move is $\mathrm{X}_{\text {new }}=\left[x_{2}, x_{3}, \ldots, x_{(M / 2)}, x_{(M / 2)+1}, x_{1}\right.$, $\left.x_{(M / 2)+2}, \ldots, x_{M}\right]$. In this research, since the initial solution is intentionally generated to have cells with a smaller number of machines than the predetermined upper limit of cell size, SWAP is not appropriate for modifying the solution. The reason for this is that SWAP cannot change the number of machines in each cell and generally cannot produce a good final solution. Therefore, INSERT is chosen for this research.

\subsection{Neighborhood Size}

The neighborhood size determines the number of neighboring solutions to be investigated at each iteration of the search process. There are two sizes of neighborhood commonly used [31]. One involves examining all neighboring solutions, and selecting the best solution that has not been tabued. This is denoted as WHLSIZE. The other involves examining neighboring solutions in order, and selecting the first non-tabued solution that improves (or equals) the current solution. When there is no such solution, the entire neighborhood will be examined, and the best non-tabued solution will be chosen. This is denoted as RANSIZE. Using the above instance, when INSERT is used to generate neighboring solutions, there will be $(M / 2)+1$ positions in each cell where we can insert a machine. The neighboring solutions of $X$ are generated as follows:

1. Insert each of the machines in cell 1 to each position in cell 2 , respectively.

2. Insert each of the machines in cell 2 to each position in cell 1 , respectively.

Since there are $(M / 2)$ machines in each cell, there will 2(M/2)[(M/2)+1] neighboring solutions for $X$ in total. If WHLSIZE is used, then all the 
neighboring solutions have to be evaluated in each iteration. While if RANSIZE is used, since it is not known, in advance, which job will be the first non-tabued solution that improves the current solution, the number of neighboring solutions to be evaluated in each iteration is random.

Note that, as mentioned above, if the best solution found in iteration is better than or equal to the incumbent solution, then the incumbent solution is updated by the best solution; otherwise, it retains the incumbent solution. In this research, the number of exceptional elements (EE) and group efficiency (GE) are considered. We have to decide how to evaluate the neighboring solutions, and how to update the incumbent solution. Since the scales of the two objectives are different, it is difficult to evaluate a solution according to both objectives. Also, according to the definitions of $\mathrm{EE}$ and GE (Section 2), the calculation of GE is more complicated than that of EE, so $\mathrm{EE}$ is used to evaluate the neighboring solutions. However, the incumbent solution will be updated only if both of the two objective values of the best non-tabued neighboring solution are better than or equal to that of the current incumbent solution. This is based on an assumption that the two objectives are equally important.

Ten test problems were used to evaluate the performance of ARTI\&TS with WHLSIZE and RANSIZE, respectively. It was found that the results generated by using WHLSIZE were slightly better than, or at least equal to, those generated by using RANSIZE. Also, the computational speed of ARTI\&TS with WHLSIZE was faster than that of ARTI\&TS with RANSIZE. Therefore, WHLSIZE is chosen for ART 1\&TS.

\subsection{Tabu List Size}

Tabu list is one of the primary features of TS. It contains a list of moves that have already been made, and are therefore forbidden or tabued. Too small a tabu list size may cause cycling of TS, while too large a tabu list size may prohibit TS from some appealing solution regions. Based on research [14], regardless of the problem size, tabu list size can be set from 5 to 12 , with 7 being the magic number. Therefore, in this study, the tabu list size chosen was seven.

\subsection{Termination Criteria}

Two termination criteria are commonly used in TS [31]: terminate if the number of iterations is greater than a maximum value given; terminate if the number of iterations without improving the best solution is greater than a specified number of iterations. Although the second criterion may be more efficient in speed, a suitable number of iterations are difficult to determine because it may be affected by the complexity of the solution space and the problem size. Therefore, the first termination rule was selected and the evolution curve of every test problem was plotted so as to observe the number of iterations required for convergence. It was found that all the curves converged within 60 iterations, but for security, the maximum number of iterations is set at 100 for ARTI\&TS.

\section{The ART1 AND TS Algorithm}

After discussing ART1 and TS, we are in the position to present the procedure of ART1\&TS. There are two parameters which have to be controlled in the algorithm: the vigilance threshold $(\rho)$ and scale coefficient $(\delta)$. In this research, the vigilance threshold $\rho$ ranges from 0.1 to 0.4 with an increment of 0.1 . The scale coefficient ranges from 0.6 and 1.0 with an increment of 0.1 . The procedure of ART1\&TS is presented in the following steps:

Step 0. Let $\mathrm{M}$ be the total number of machines and $m$ be the upper limit of cell size.

Step 1. Set the scale coefficient $\delta=1.0$.

Step 2. Decide the maximum number of cells (number of output neurons).

$$
T C=\operatorname{Int}\left(\frac{M}{m \cdot \delta}\right)+1
$$

Step 3. Set the vigilance threshold $\rho=0.1$.

Step 4. Perform the Modified ART1 with the given $\delta$ and $\rho$ values to generate the initial cell formation.

Step 5. Assign each of the nonclustered machines to the output neuron that will result in the smallest number of exceptional elements.

Step 6. Refine the solution produced in Step 5 using the basic TS heuristic.

Step 7. If $\rho=\rho+0.1<0.5$, go to Step 4; otherwise, go to Step 8 .

Step 8. If $\delta=\delta-0.1>0.5$, go to Step 2; otherwise, stop.

\section{COMPUTATIONAL RESULTS}

In this research, thirty-one popular test problems were used to evaluate the performance of ART1\&TS. Table 1 displays a list of the test problems in an ascending order of their problem sizes (number of machines). The data include the number of machines (M), number of parts (N), and source for each problem. The ART1\&TS program was coded in $\mathrm{C}$ language and run on an IRIX System, Release 5.3.

For each test problem, in order to perform a fair comparison between the solution produced by ART1\&TS and the best existing solution, the number 
of cells (NG) in the existing solution was used as a constraint while implementing ART1\&TS. Three values were generated by $\operatorname{Int}(\mathrm{M} / \mathrm{NG})$ plus one, two and three, respectively, and each value was used as an upper limit of cell size, $\mathrm{m}$, in ARTI\&TS. Where Int(M/NG) stands for rounding down the answer of $\mathrm{M} / \mathrm{NG}$ to the nearest integer. Since different threshold values $(\rho)$ and scale coefficients $(\delta)$ were used, different solutions were produced for each $\mathrm{m}$ value. However, as mentioned above, only the solutions with the same number of cells as the specified NG value were considered. Note that the determination of the three mvalues, and the ranges of threshold values, $0.1 \sim 0.4$, and scale coefficients, 0.6 1.0, was performed by conducting a thorough experiment on the first ten test problems. In the experiment, the same range, $0.1 \sim 1.0$, was used for both threshold value and scale coefficient.

Table 2 presents the range combination of threshold values and scale coefficients (for each $\mathrm{m}$ value in each test problem) where the ART1\&TS generated the best solution under the constraint of the specified NG value. The symbol, -----, in the entries of threshold value and scale coefficient of a test problem, refers to a condition where the best solution found did not have the same number of cells as its corresponding NG value. It is clear that the range combination of threshold values and scale coefficients for generating the best solution varies for each problem. In examining all these combinations, it was found that the combination of 0.3 0.4 $(\rho)$ and 0.7-0.6 $(\delta)$ solved most of the problems, except problems 14 , $19,20,26$, and 29. These five problems could be solved by the combination of $0.1 \sim 0.4(\rho)$ and $0.7 \sim 0.6$ $(\delta)$. This is an expected result. That is, using a medium to low threshold value and a medium scale coefficient, the Modified ART1 may assign more machines in a cell, but will not fill up the cell. For an initial solution that includes a reasonable number of cells, with the machines in each cell containing a certain degree of similarity, the implementation of TS can more effectively improve the solution.

The results in Table 2 also reveal the effect of scaling the upper limit of cell size on the performance of ART 1\&TS. In the first twenty problems, except problems $11,13,14,18$, the best solutions could be produced with $\delta=1.0$. That is, the upper limit of cell size was not scaled while implementing the Modified ART1, and the best solution could still be generated by TS. However, in the last eleven problems, none of the best solutions were generated by ART1\&TS with $\delta=1.0$. Note that these problems were listed in ascending order of problem size. Therefore, it is concluded that this scaling procedure is a critical factor in the performance of ART1\&TS for larger size problems.

A comparison between the solution generated by ART1\&TS, and the existing best solution for each test problem, is presented in Table 3. Since in several problems a different number of cells (NG) were considered, this comparison is based on all different cases (forty-one). Also, in order to evaluate the effect of the Modified ART1 on the performance of ART1\&TS, the solution generated by TS with a random initial solution (denoted as RAN\&TS) is also presented. It was discovered that of the forty-one cases, ART1\&TS produces the same best solutions in twenty-eight cases, and dominates the existing best solutions in nine cases. In the remaining four cases, ART 1\&TS produced all the solutions with a smaller number of exceptional elements, but with slightly worse group efficiency. The symbols, * and !, in Table 3 represent these nine and four cases, respectively. It is noteworthy that most of these test problems have been developed and solved by several different heuristics over the years, so the existing best solutions for the problems should be promising. Therefore, it is concluded that ART1\&TS is an efficient heuristic for cell formation problems when the number of exceptional elements and group efficiency are considered as the objectives.

The comparison between ART1\&TS and RAN\&TS shows that they are equal in thirty-two cases, but ART1\&TS exceeds RAN\&TS in the remaining nine cases. Furthermore, six of the nine cases appear in the last ten problems. This result is not surprising because TS has been proven to be an effective optimization technique. For the problem with a smaller size, or less complicated objective function, it is usually able to generate a good final solution regardless of the initial solution. However, the effect of the initial solution becomes significant if the problem size is large, or the objective function is complicated. This is the advantage of ART 1\&TS compared with RAN\&TS. The symbol, $\perp$, in the table represents the condition where ART1\&TS outperforms RAN\&TS. A further observation of the effect of an initial solution on ART1\&TS was conducted by applying ART $1 \&$ TS and RAN\&TS to a larger size problem $(60 \times 540)$ cited from Chen et al. [10]. Since group efficiency was not presented for the problems, where simulated annealing (SA) was the heuristic used, the number of exceptional elements was used to evaluate the performance of ART1\&TS, RAN\&TS, and SA. Table 4 shows that ART1\&TS outperforms both RAN\&TS and SA, especially, when the upper limit of cell size is smaller. This may result from the fact that the smaller the limit, usually, the larger the number of cells, and the more complicated the objective function. This result strongly confirms the effect of the Modified ART1 on the performance of ART1\&TS.

There are two other points deserving mention. First, two important factors of cell formation problems, number of cells and upper limit of cell size, 
Table 1. A list of test problems

\begin{tabular}{|c|c|c|c|}
\hline $\begin{array}{l}\text { Problem } \\
\text { Number }\end{array}$ & $\begin{array}{c}\text { Machines } \\
\text { (M) }\end{array}$ & $\begin{array}{l}\text { Parts } \\
(\mathrm{N})\end{array}$ & Source \\
\hline 1 & 5 & 7 & King and Nakornchai (1982) \\
\hline 2 & 5 & 18 & Seifoddini (1982) \\
\hline 3 & 6 & 8 & Kusiak and Cho (1992) \\
\hline 4 & 7 & 8 & Kusiak and Chow (1987) \\
\hline 5 & 7 & 11 & Kusiak and Chow (1987) \\
\hline 6 & 7 & 11 & Boctor (1991) \\
\hline 7 & 8 & 12 & Seifoddini and Wolfe (1986) \\
\hline 8 & 8 & 20 & Chandrasekharan and Rajagopalan (1986) \\
\hline 9 & 10 & 10 & Mosier and Taube (1985) \\
\hline 10 & 10 & 15 & Askin et al. (1991) \\
\hline 11 & 12 & 19 & De Witte (1980) \\
\hline 12 & 14 & 24 & Stanfel $(1985)$ \\
\hline 13 & 14 & 43 & Burbidge (1975) excluding machines 6 and 8 \\
\hline 14 & 16 & 30 & Srinivasan et al. (1990) \\
\hline 15 & 16 & 30 & Boctor (1991, data set1) \\
\hline 16 & 16 & 30 & Boctor (1991, data set2) \\
\hline 17 & 16 & 30 & Boctor (1991, data set3) \\
\hline 18 & 16 & 30 & Boctor (1991, data set4) \\
\hline 19 & 16 & 30 & Boctor (1991, data set5) \\
\hline 20 & 16 & 43 & Burbidge (1975) \\
\hline 21 & 20 & 20 & Harhalskis et al. (1990) \\
\hline 22 & 20 & 35 & Carrie (1973) \\
\hline 23 & 20 & 35 & Boe and Cheng (1991) \\
\hline 24 & 22 & 43 & Burbidge (1975) \\
\hline 25 & 24 & 40 & Chandrasekharan and Rajagopalan(1989, data set 1) \\
\hline 26 & 24 & 40 & Chandrasekharan and Rajagopalan(1989, data set 2) \\
\hline 27 & 24 & 40 & Chandrasekharan and Rajagopalan(1989, data set 3) \\
\hline 28 & 24 & 40 & Chandrasekharan and Rajagopalan $(1989$, data set 4$)$ \\
\hline 29 & 24 & 40 & Chandrasekharan and Rajagopalan(1989, data set 7) \\
\hline 30 & 30 & 41 & Kumar and Vannelli (1987) \\
\hline 31 & 40 & 100 & Chandrasekharan and Rajagopalan(1987) \\
\hline
\end{tabular}

Table 2. The condition where ART1 and TS produces the best solution

\begin{tabular}{|c|c|c|c|c|c|c|c|c|c|}
\hline $\begin{array}{l}\text { Problem } \\
\text { Number }\end{array}$ & NG & $\mathrm{m}$ & $\rho$ & $\delta$ & $\begin{array}{l}\text { Problem } \\
\text { Number }\end{array}$ & NG & $\mathrm{m}$ & $\rho$ & $\delta$ \\
\hline \multirow[t]{3}{*}{1} & 2 & 3 & $0.1 \sim 0.4$ & $1.0 \sim 0.6$ & 10 & 3 & 4 & $0.1 \sim 0.4$ & $1.0 \sim 0.6$ \\
\hline & & 4 & $0.1 \sim 0.4$ & $1.0 \sim 0.6$ & & & 5 & $0.1 \sim 0.3$ & $0.9 \sim 0.8$ \\
\hline & & 5 & $0.1 \sim 0.4$ & $1.0 \sim 0.6$ & & & & 0.4 & $0.9 \sim 0.7$ \\
\hline \multirow[t]{3}{*}{2} & 2 & 3 & $0.1 \sim 0.4$ & 1 & & & & 0.3 & 0.6 \\
\hline & & 4 & $0.1 \sim 0.4$ & $1.0 \sim 0.6$ & & & 6 & 0.3 & 0.6 \\
\hline & & 5 & --- & --- & 11 & & 3 & 5 & $0.1 \sim 0.3$ \\
\hline \multirow[t]{3}{*}{3} & 2 & 4 & $0.1 \sim 0.4$ & $0.9 \sim 0.6$ & & & & & 0.4 \\
\hline & & 5 & $0.1 \sim 0.4$ & $1.0 \sim 0.6$ & & & & 6 & $0.3 \sim 0.4$ \\
\hline & & 6 & --- & --- & & & & 7 & --- \\
\hline \multirow[t]{3}{*}{4} & 3 & 3 & $0.1 \sim 0.4$ & $1.0 \sim 0.6$ & 12 & & 4 & 4 & $0.1 \sim 0.4$ \\
\hline & & 4 & $0.1 \sim 0.4$ & $1.0 \sim 0.6$ & & & & 5 & $0.1 \sim 0.4$ \\
\hline & & 5 & $0.1 \sim 0.4$ & $0.8 \sim 0.6$ & & & & 6 & $0.1 \sim 0.4$ \\
\hline \multirow[t]{3}{*}{5} & 2 & 4 & $0.1 \sim 0.4$ & $1.0 \sim 0.6$ & 13 & & 5 & 3 & --- \\
\hline & & 5 & $0.1 \sim 0.4$ & $1.0 \sim 0.6$ & & & & 4 & $0.1 \sim 0.4$ \\
\hline & & 6 & $0.1 \sim 0.4$ & $0.8 \sim 0.6$ & & & & 5 & $0.1 \sim 0.4$ \\
\hline
\end{tabular}


Table 2. (continuous)

\begin{tabular}{|c|c|c|c|c|c|c|c|c|}
\hline 6 & 3 & 3 & $0.1 \sim 0.4$ & $1.0 \sim 0.6$ & 14 & 4 & 3 & --- \\
\hline & & 4 & $0.1 \sim 0.3$ & $0.9 \sim 0.8$ & & & 4 & 0.1 \\
\hline & & & 0.4 & $0.9 \sim 0.6$ & & & 5 & 0.1 \\
\hline & & 5 & 0.4 & $0.7 \sim 0.6$ & 15 & 2 & 9 & $0.1 \sim 0.4$ \\
\hline \multirow[t]{3}{*}{7} & 3 & 3 & $0.1 \sim 0.4$ & $1.0 \sim 0.7$ & & & 10 & $0.1 \sim 0.4$ \\
\hline & & 4 & --- & --- & & & 11 & $0.1 \sim 0.4$ \\
\hline & & 5 & --- & --- & & 3 & 6 & $0.1 \sim 0.4$ \\
\hline \multirow[t]{6}{*}{8} & 2 & 5 & --- & --- & & & 7 & --- \\
\hline & & 6 & $0.1 \sim 0.4$ & $1.0 \sim 0.7$ & & & 8 & --- \\
\hline & & 7 & $0.1 \sim 0.4$ & $0.7 \sim 0.6$ & & 4 & 5 & --- \\
\hline & 3 & 3 & \begin{tabular}{l|}
-- \\
\end{tabular} & --- & & & 6 & 0.4 \\
\hline & & 4 & $0.1 \sim 0.4$ & $0.9 \sim 0.6$ & & & 7 & --- \\
\hline & & 5 & --- & --- & 16 & 3 & 6 & $0.1 \sim 0.4$ \\
\hline \multirow[t]{3}{*}{9} & 3 & 4 & $0.1 \sim 0.4$ & $1.0 \sim 0.6$ & & & 7 & $0.1 \sim 0.4$ \\
\hline & & 5 & $0.1 \sim 0.4$ & $1.0 \sim 0.6$ & & & 8 & $0.1 \sim 0.4$ \\
\hline & & 6 & $0.1 \sim 0.4$ & $0.9 \sim 0.6$ & & & & \\
\hline \multirow[t]{6}{*}{17} & & 3 & 6 & $0.1 \sim 0.4$ & 23 & 4 & 6 & $0.1 \sim 0.4$ \\
\hline & & & 7 & --- & & & 7 & $0.1 \sim 0.4$ \\
\hline & & & 8 & --- & & & 8 & --- \\
\hline & & 4 & 4 & $0.1 \sim 0.4$ & 24 & 5 & 5 & --- \\
\hline & & & 5 & $0.1 \sim 0.4$ & & & 6 & $0.1 \sim 0.4$ \\
\hline & & & 6 & $0.1 \sim 0.4$ & & & 7 & $0.1 \sim 0.4$ \\
\hline \multirow[t]{3}{*}{18} & & 3 & 6 & --- & 25 & 7 & 4 & --- \\
\hline & & & 7 & 0.4 & & & 5 & 0.1 \\
\hline & & & 8 & 0.4 & & & 6 & 0.1 \\
\hline \multirow[t]{4}{*}{19} & & 3 & 6 & --- & 26 & 7 & 4 & --- \\
\hline & & & 7 & $0.1 \sim 0.2$ & & & 5 & 0.1 \\
\hline & & & & 0.4 & & & 6 & 0.1 \\
\hline & & & 8 & 0.4 & 27 & 7 & 4 & --- \\
\hline \multirow[t]{11}{*}{20} & & 3 & 6 & $0.1 \sim 0.2$ & & & 5 & $0.1 \sim 0.3$ \\
\hline & & & & $0.3 \sim 0.4$ & & & 6 & $0.2 \sim 0.3$ \\
\hline & & & 7 & --- & 28 & 7 & 4 & --- \\
\hline & & & 8 & --- & & & 5 & $0.1 \sim 0.3$ \\
\hline & & 4 & 5 & $0.1 \sim 0.4$ & & & 6 & $0.1 \sim 0.3$ \\
\hline & & & 6 & --- & 29 & 9 & 3 & 0.1 \\
\hline & & & 7 & --- & & & 4 & --- \\
\hline & & 5 & 4 & --- & & & 5 & --- \\
\hline & & & 5 & $0.1 \sim 0.2$ & 30 & 4 & 8 & --- \\
\hline & & & & $0.3 \sim 0.4$ & & & 9 & 0.4 \\
\hline & & & 6 & 0.1 & & & 10 & --- \\
\hline \multirow[t]{6}{*}{21} & & 4 & 6 & --- & & 5 & 7 & --- \\
\hline & & & 7 & $0.1 \sim 0.4$ & & & 8 & --- \\
\hline & & & 8 & $0.3 \sim 0.4$ & & & 9 & 0.3 \\
\hline & & 5 & 5 & $0.1 \sim 0.4$ & 31 & 10 & 5 & --- \\
\hline & & & 6 & $0.1 \sim 0.4$ & & & 6 & 0.1 \\
\hline & & & 7 & $0.1 \sim 0.4$ & & & 7 & 0.4 \\
\hline \multirow[t]{3}{*}{22} & & 4 & 6 & $0.1 \sim 0.4$ & & & & \\
\hline & & & 7 & $0.1 \sim 0.4$ & & & & \\
\hline & & & 8 & $0.1 \sim 0.4$ & & & & \\
\hline
\end{tabular}


Table 3. A comparison of the performance of existing methods, RAN \& TS and ART \& TS

\begin{tabular}{|c|c|c|c|c|c|c|c|c|c|c|c|}
\hline \multirow[b]{2}{*}{ NO } & \multirow[b]{2}{*}{ Reference } & \multicolumn{3}{|c|}{ Existing Solution } & \multicolumn{3}{|c|}{ RAN \& TS } & \multicolumn{4}{|c|}{ ART1 \& TS } \\
\hline & & NG & $\mathrm{EE}$ & GE & NG & $\mathrm{EE}$ & GE & NG & $\mathrm{EE}$ & GE & $\begin{array}{l}\text { CPU } \\
(\mathrm{Sec})\end{array}$ \\
\hline \multirow[t]{2}{*}{1} & King et al. (1982) & 2 & 0 & 0.91 & 2 & 0 & 0.91 & 2 & 0 & 0.91 & 0.04 \\
\hline & Chen and Cheng (1995) & & & & & & & & & & \\
\hline \multirow[t]{2}{*}{2} & Seifoddini (1989) & 2 & 5 & 0.87 & 2 & 5 & 0.87 & 2 & 5 & 0.87 & 0.10 \\
\hline & Chen and Cheng(1995) & 2 & 7 & 0.89 & 2 & 7 & 0.89 & 2 & 7 & 0.89 & 0.11 \\
\hline 3 & Kusiak and Cho(1992) & 2 & 2 & 0.88 & 2 & 2 & 0.88 & 2 & 2 & 0.88 & 0.07 \\
\hline 4 & Kusiak and Cho(1987) & 3 & 0 & 0.83 & 3 & 0 & 0.83 & 3 & 0 & 0.83 & 0.07 \\
\hline 5 & Chen and Cheng(1995) & 2 & 3 & 0.73 & 2 & 3 & 0.73 & 2 & 3 & 0.73 & 0.08 \\
\hline 6 & Mukhopadhyay et al.(1995) & 3 & 2 & 0.86 & 3 & 2 & 0.86 & 3 & 2 & 0.86 & 0.15 \\
\hline 7 & Seifoddini et al.(1986) & 3 & 7 & 0.86 & 3 & 7 & 0.86 & 3 & 7 & 0.86 & 0.15 \\
\hline \multirow[t]{3}{*}{8} & Chu and Tsai(1990) & 2 & 5 & 0.80 & 2 & 5 & 0.80 & 2 & 5 & 0.80 & 0.29 \\
\hline & Chandrasekharan and & 3 & 9 & 0.86 & 3 & 9 & 0.86 & 3 & 9 & 0.86 & 0.25 \\
\hline & Rajagopalan(1986) & & & & & & & & & & \\
\hline 9 & Mukhopadhyay et al.(1995) & 3 & 0 & 0.85 & 3 & 0 & 0.85 & 3 & 0 & 0.85 & 0.15 \\
\hline 10 & Askin et al.(1991) & 3 & 5 & 0.92 & 3 & 5 & 0.92 & 3 & 5 & 0.92 & 0.51 \\
\hline \multirow[t]{2}{*}{11} & Chen and Cheng(1995) & 3 & 21 & 0.771 & & & & & & & \\
\hline & Chen and Cheng(1995) & $* 3$ & 20 & 0.767 & 3 & 17 & 0.777 & 3 & 17 & 0.777 & 0.95 \\
\hline 12 & Askin et al.(1991) & 4 & 2 & 0.84 & 4 & 2 & 0.84 & 4 & 2 & 0.84 & 1.23 \\
\hline 13 & Askin et al.(1991) & 5 & 3 & 0.83 & 5 & 3 & 0.83 & 5 & 3 & 0.83 & 1.08 \\
\hline 14 & Chen and Cheng(1995) & 4 & 19 & 0.86 & $\perp 4$ & 25 & 0.84 & 4 & 19 & 0.86 & 2.06 \\
\hline \multirow{3}{*}{15} & Mukhopadhyay et al.(1995) & $* 2$ & 33 & 0.61 & 2 & 11 & 0.71 & 2 & 11 & 0.71 & 1.06 \\
\hline & & $* 3$ & 30 & 0.72 & 3 & 30 & 0.73 & 3 & 30 & 0.73 & 1.17 \\
\hline & & $! 4$ & 31 & 0.76 & $\perp 4$ & 36 & 0.74 & 4 & 29 & 0.74 & 2.10 \\
\hline 16 & Mukhopadhyay et al.(1995) & $! 3$ & 11 & 0.87 & 3 & 7 & 0.80 & 3 & 7 & 0.80 & 2.32 \\
\hline \multirow[t]{2}{*}{17} & Mukhopadhyay et al.(1995) & $* 3$ & 15 & 0.71 & 3 & 11 & 0.74 & 3 & 11 & 0.74 & 1.58 \\
\hline & & $! 4$ & 11 & 0.87 & 4 & 8 & 0.86 & 4 & 8 & 0.86 & 1.39 \\
\hline 18 & Mukhopadhyay et al.(1995) & 3 & 23 & 0.72 & 3 & 23 & 0.72 & 3 & 23 & 0.72 & 1.62 \\
\hline \multirow[t]{2}{*}{19} & Mukhopadhyay et al.(1995) & $* 3$ & 22 & 0.69 & 3 & 9 & 0.79 & 3 & 9 & 0.79 & 2.54 \\
\hline & & 4 & 11 & 0.88 & 4 & 11 & 0.88 & 4 & 11 & 0.88 & 1.94 \\
\hline \multirow[t]{4}{*}{20} & Mukhopadhyay et al.(1995) & $* 3$ & 30 & 0.66 & $\perp 3$ & 25 & 0.69 & 3 & 22 & 0.70 & 2.32 \\
\hline & Boctor $(1991)$ & $* 4$ & 26 & 0.75 & 4 & 26 & 0.76 & 4 & 26 & 0.76 & 1.85 \\
\hline & Askin et al. (1991) & $! 5$ & 29 & 0.80 & - & - & - & - & - & - & - \\
\hline & Chen and Cheng(1995) & 5 & 27 & 0.79 & 5 & 27 & 0.79 & 5 & 27 & 0.79 & 1.91 \\
\hline \multirow[t]{2}{*}{21} & Chen and Cheng(1995) & 4 & 11 & 0.81 & $\perp 4$ & 13 & 0.79 & 4 & 11 & 0.81 & 3.85 \\
\hline & Chen and Cheng(1995) & 5 & 14 & 0.89 & 5 & 14 & 0.89 & 5 & 14 & 0.89 & 3.44 \\
\hline 22 & Chen and Cheng(1995) & 4 & 2 & 0.88 & 4 & 2 & 0.88 & 4 & 2 & 0.88 & 3.55 \\
\hline 23 & Chen and Cheng(1995) & 4 & 34 & 0.80 & 4 & 34 & 0.80 & 4 & 34 & 0.80 & 5.21 \\
\hline 24 & Kusiak and Cho(1992) & 5 & 3 & 0.81 & 5 & 3 & 0.81 & 5 & 3 & 0.81 & 3.71 \\
\hline \multirow[t]{2}{*}{25} & Chandrasekharan and & 7 & 0 & 1.00 & 7 & 0 & 1.00 & 7 & 0 & 1.00 & 4.80 \\
\hline & Rajagopalan(1989) & & & & & & & & & & \\
\hline \multirow[t]{2}{*}{26} & Chandrasekharan and & 7 & 10 & 0.95 & $\perp 7$ & 14 & 0.95 & 7 & 10 & 0.95 & 7.95 \\
\hline & Rajagopalan(1989) & & & & & & & & & & \\
\hline \multirow[t]{2}{*}{27} & Chandrasekharan and & 7 & 20 & 0.91 & 7 & 20 & 0.91 & 7 & 20 & 0.91 & 9.55 \\
\hline & Rajagopalan(1989) & & & & & & & & & & \\
\hline 28 & Chandrasekharan and & $* 7$ & 20 & 0.85 & 7 & 19 & 0.91 & 7 & 19 & 0.91 & 9.44 \\
\hline & Rajagopalan(1989) & & & & & & & & & & \\
\hline 29 & Srinivasan et al. (1991) & $* 9$ & 61 & 0.79 & $\perp 9$ & 60 & 0.79 & 9 & 58 & 0.80 & 5.34 \\
\hline 30 & Chen and Cheng(1995) & 4 & 8 & 0.69 & $\perp 4$ & 10 & 0.68 & 4 & 8 & 0.69 & 7.51 \\
\hline & Chen and Cheng(1995) & 5 & 10 & 0.72 & $\perp 5$ & 20 & 0.71 & 5 & 10 & 0.72 & 7.76 \\
\hline 31 & Chandrasekharan and & 10 & 36 & 0.95 & $\perp 10$ & 52 & 0.87 & 10 & 36 & 0.95 & 68.07 \\
\hline & Rajagopalan(1987) & & & & & & & & & & \\
\hline
\end{tabular}

Note: NG: number of cells; EE: number of exceptional elements; Ge: grouping efficiency

*: ART1 \& TS solution dominates the best existing solution

! : ART1 \& TS solution has better EE, but worse GE than the best existing solution

$\perp$ : ART1 \& TS solution dominates RAN \& TS solution 
Table 4. A comparison of the performance of SA, RAN\&TS, and ART1\&TS

\begin{tabular}{|c|c|c|c|c|c|}
\hline Problem & Cell size & SA Solution & RAN\&TS & ART1\&TS & CPU(sec) \\
\hline $60 \times 540$ & 8 & 810 & 832 & 796 & 296.35 \\
\hline & 9 & 675 & 666 & 638 & 162.83 \\
\hline & 10 & 490 & 481 & 458 & 117.47 \\
\hline & 11 & 305 & 288 & 286 & 115.17 \\
\hline & 12 & 117 & 117 & 117 & 53.82 \\
\hline & 13 & 117 & 117 & 117 & 124.36 \\
\hline
\end{tabular}

can be taken into account while implementing ART 1\&TS. This makes ART1\&TS a more practical tool than all other applications of ART1for cell formation problems. Second, the computational speed of ART1\&TS is quite efficient. Generally, the CPU time for solving a cell formation problem is affected by the size and structure of the problem. Due to the TS procedure of ART1\&TS, it is also affected by the specified number of cells (NG). Table 3 shows that the CPU times for the first ten problems are less than one second; the second ten problems, three seconds, and third ten problems, ten seconds. For the last problem, $40 \times 100$ and 10 cells, the CPU time is 68.07 seconds. Also, in Table 4, for the problem, 60 $\times 540$, with the constraint of different upper limits of cell size, the CPU times vary from 53.82 to 296.35 seconds. These results indicate that ART1\&TS is still efficient even for large size problems.

\section{CONCLUSIONS}

In this paper, a new approach (ART 1\&TS), integrating Modified ART 1 and TS, has been developed for cell formation problems with the number of exceptional elements (EE) and group efficiency (GE) as objectives. With the following designs in ART1\&TS, the computational results showed that it produced the best solutions in most of the test problems with very reasonable computational speed. First, while implementing Modified ART1 to cell formation problems, the upper limit of cell size is scaled, and the number of output neurons is limited. This usually generated good initial solutions that were effectively and efficiently improved by TS. The results indicated that this design was especially critical for large size problems. Second, using EE as the criterion for evaluating the neighboring solutions in iteration, the best non-tabued neighboring solution is then selected as the move to transfer from the current iteration to the next iteration. Since the calculation of the change of EE in a move is trivial, the implementation of TS becomes very efficient.

Furthermore, since A RT1\&TS can consider both the number of cells and the upper limit of cell size as the constraints when solving cell formation problems, this makes it a good tool for practical problems .

\section{REFERENCES}

1. Askin, R. G., S. H. Cresswell, J. B. Goldberg and A. J. Vkhariaa, "A hamiltonian path approach to reordering the part-machine matrix for cellular manufacturing," International Journal of Production Research, 29, 1081-1100 (1991).

2. Boctor, F. F., "A liner formulation of the machine-part cell formation," International Journal of Production Research, 29, 343-356 (1991).

3. Boe, W. J. and C. H. Cheng, "A close neighbor algorithm for designing cellular manufacturing systems," International Journal of Production Research, 29, 2097-2116 (1991).

4. Buridge, J. L., The introduction of Group Technology, John Wiley, New York (1975).

5. Carp enter, G. A. and S. Grossberg, "Neural dynamics of category learning and recognition: attention, memory consolidation and amnesia," in Brain Structure, Learning and memory, J. L. Davis, R. W. Newbrugh, and E. J. Wegman (eds.), AAAS Symposium series, Boulder, Colorado (1986).

6. Carrie, A. S., "Numerical taxonomy applied to group technology and plant layout," International Journal of Production Research, 11, 399-416 (1973).

7. Chandrasekharan, M. P. and R. Rajagopalan, "An ideal seed non-hierarchical clustering algorithm for cellular manufacturing," International Journal of Production Research, 24, 451-464 (1986).

8. Chandrasekharan, M. P. and R. Rajagopalan, "ZODIAK-an algorithm for concurrent formation of part-families and machine-cells," International Journal of Production Research, 25, 835-850(1987).

9. Chandrasekharan, M. P. and R. Rajagopalan, "Groupability: an analysis of the properties of binary data matrices for group technology," International Journal of Production Research, 27, 1035-1052 (1989).

10. Chen, C. L., N. A. Cotmvo and W. Baek, "A simulated annealing solution to the cell formation problem," International Journal of Production Research, 33, 2601-2614 (1995).

11. Chen, S. J. and C. S. Cheng, "A neural network-based cell formation algorithm in cellular manufacturing," International Journal of Production Research, 33, 293-318 (1995).

12. Dagli, C. and R. Huggahalli, "Neural network approach to group technology," in Knowledge Based Systems and Neural Networks: Techniques and 
Applications, R. Sharda, J. Y. Cheung, W. J. Cochran, S. Oklahoma (eds.), Elsevier, New York (1991).

13. De Witte, J., "The use of similarity coefficients in production flow analysis," International Journal of Production Research, 18, 503-514 (1980).

14. Glover, F., "Tabu search. part I," ORSA Journal on Computing, 1, 190-206 (1989).

15. Glover, F. and M. Laguna, "Tabu Search," in Morden Heuristic Techniques for Combinatorial, C. R. Reeves (ed.), Blackwell Scientific, Oxford (1993).

16. Harhalakis, G., R. Nagi and J. M. Proth, "An efficient heuristic in manufacturing cell formation for group technology applications," International Journal of Production Research, 28, 185-198 (1990).

17. Heragu, S., "Group technology and cellular manufacturing," IEEE Transactions on Systems, Man, and Cybernetics, 24, 203-214 (1994).

18. Kaparthi, S. and N. C. Suresh, "Machine-component cell formation in group technology: a neural network approach," International Journal of Production Research, 30, 1353-1367 (1992).

19. Kaparthi, S., N. C. Suresh and R. P. Cerveny, "An improved neural network leader algorithm for part-machine grouping in group technology," European Journal of Operational Research, 69, 342-356 (1993).

20. King, J. R. and V. Nakornchai, "Machine component group formation in group technology--review and extension," International Journal of Production Research, 20, 117-133 (1982).

21. Kusiak, A. and M. Cho, "Similarity coefficient algorithms for solving the group technology problem," International Journal of Production Research, 22, 937-948 (1992).

22. Kusiak, A. and W. S. Chow, "Efficient solving of the group technology problem," Journal of Manufacturing Systems, 6, 117-124 (1987)

23. Mosier, C. and L. Taube, "The facets of group technology and their impact on implementation: a state-of-the-art survey," Omega, 13, 381-389(1985).

24. Rao, H. A. and P. Gu, "Design of cellular manufacturing systems: a neural net approach," International Journal of System Automation: Research and Applications, 2, 407-424 (1992).

25. Seifoddini, H., "A note on the similarity coefficient method and the problem of improper machine assignment in group technology applications," International Journal of Production Research, 27, 1161-1165 (1989).

26. Seifoddini, H. and P. M. Wolfe, "Application of the similarity coefficient method in group technology," IIE Transactions, 18, 271-277 (1986).

27. Singh, N., "Design of cellular manufacturing systems: an invited review," European Journal of Operational Research, 69, 284-291 (1993).

28. Srinivasan, G., T. T. Narendran and B. Mahadevan, "Assignment model for the part-families problem in group technology," International Journal of
Production Research, 28, 145-152 (1990).

29. Stanfel, L. E., "Machine clustering for economic production," Engineering Costs and Production Economics, 9, 73-81 (1985).

30. Taillard, E., "Some efficient heuristic methods for the flow shop sequencing problem," European Journal of Operational Research, 47, 75-82 (1990).

31. Widmer, M. and A. Hertz, "A new heuristic method for the flow shop sequencing problem," European Journal of Operational Research, 41, 186-193(1989).

\section{ABOUT THE AUTHORS}

Ming-Laing Chen is the manager of Management Information Department at Master Design Corporation, California USA. He holds MS and $\mathrm{Ph} . \mathrm{D}$. degrees from Mississippi State University. His research interests are production control systems, and optimization methods.

Chung-Min Wu received his B.S. degree in Industrial Engineering from Chuan-Yuan University, Taiwan, in 1981. Subsequently, he received his Master and Ph.D. degree in Industrial Engineering in 1986 and 1991, respectively, from Auburn University, Alabama USA. He is currently an associate professor in Department of Business Management at National Taipei University of Technology. His research interests include data mining, evolutionary computation, pattern recognition, and artificial neural networks.

Chuen-Lung Chen is an associate professor in Department of Management Information Systems at National Chengchi University. He received his Ph.D. degree from Auburn University, Alabama USA. He was previously a faculty member in the Industrial Engineering Department at Mississippi State University. His research interests include production control systems, optimization methods, and supply chain management.

(Received December 2000; revised July 2001; accepted December 2001) 


\title{
結合自適應共振理論網路和禁忌搜尋法求解群組構成問題
}

\author{
陳明良 \\ 密西西比州立大學工業工程系 \\ 吳忠敏 ${ }^{*}$ \\ 國立台北科技大學經營管理系 \\ 106 台北市忠孝東路三段一號 \\ 陳春龍 \\ 國立政治大學資訊管理學系
}

摘要

\begin{abstract}
自適應共振理論網路 (ART1) 是類神經網路應用在群組構成問題中相當普遍的一種 網路。最近有一些研究已針對自適應共振理論網路 (ART1) 在這方面的應用提出改 善的方法。在本研究中, 我們結合一種修正的自適應共振理論網路 (ART1) 與最佳 化方法-禁忌搜尋法 (Tabu search) -來求解群組構成問題。我們考虑的群組構成問題 是在群組數目與群組大小的限制下同時達到組間移動數目最小 ( $\mathrm{EE})$ 與分群效率 (GE) 最高的目標。本方法 (ART1 \& TS) 首先使用所選定的修正自適應共振理論網路 (ART1) 產生起始的群組解，再利用禁忌搜尋法 (Tabu search) 來改善該起始解。 為了評量本方法的效能, 我們利用它來求解過去研究所使用的大部分例題。計算的結 果顯示本方法在大部分的例題中都能產生最佳的解。
\end{abstract}

關鍵詞：自適應共振理論網路，群組構成，禁忌搜尋法

(連絡人: cmwu@ntut.edu.tw) 